\title{
Wertschätzung der Mitarbeiter durch Führungskräfte
}

\author{
Paul Fuchs-Frohnhofen
}

Online publiziert: 24. Oktober 2012

(C) Springer-Verlag Wien 2012

\section{Hintergrund}

Die hier dargestellten Ergebnisse entstammen den Projekten PflegeWert (www.pflegeWert.info) und Gender-Med-AC (http://www.mat-gmbh.de/gendermned.html). In den Evaluationsarbeiten beider Projekte ist deutlich geworden, dass Führungskräfte sehr unterschiedlichen und teils gegensätzlichen Anforderungen gerecht werden müssen. Sie müssen flexibel sein, so dass sie ihr Verhalten und ihren Führungsstil auf die individuellen Mitarbeitenden anpassen können. Gleichzeitig müssen sie eine klare Linie finden und zeigen, Orientierung geben und die Richtung bestimmen. Diese unterschiedlichen Führungsrollen zu vereinen, ist auch für erfahrene Führungskräfte eine stetige Herausforderung. Im Bereich der Pflege wird diese Herausforderung häufig dadurch verstärkt, dass Themen wie Führung und Führungsstile, Projektleitung und ähnliches weder Bestandteil der Ausbildung noch der Tätigkeit selbst sind.

Der Fachkräftemangel in der Pflege erhöht gleichzeitig die Anforderungen an die Einrichtungen, durch gute Führungskräfte den Mitarbeitenden gegenüber Wertschätzung zu zeigen, um Mitarbeitende zu binden und zu motivieren, als Botschafter der jeweiligen Einrichtung in ihrem Umfeld zur Gewinnung neuer Fachkräfte beizutragen.

\section{Methoden}

Die Ergebnisse beruhen auf der Auswertung von Mitarbeiterbefragungen, der Einbindung von pflegenden Führungskräften in Entwicklungsteams und der Entwicklung,
Durchführung und Evaluation von prototypischen Testseminaren zum Thema „Wertschätzende Führung“.

\section{Ergebnisse und Schlussfolgerung}

Die in den beiden Projekten entwickelten Seminarkonzepte bieten Führungskräften - neben theoretischen Inhalten zu Führung und Wertschätzung - die Möglichkeit, das eigene Führungsverhalten zu reflektieren und wertschätzende Verhaltensweisen in teils schwierigen Situationen zu erproben. Es wird deutlich, dass sich gute Führung nur in einer wertschätzenden Unternehmenskultur entwickeln kann, die auf fachliche Kompetenz genauso setzt wie auf die Wertschätzung der Mitarbeitenden sowohl in ihrer kundenbezogenen Arbeit als auch in ihrer persönlichen Situation z. B. bzgl. der Vereinbarkeit von Familie und Beruf. Um Wertschätzung geben zu können, müssen Führungskräfte auch lernen, sich selbst, ihre Leistung, aber auch ihre persönlichen Bedürfnisse besser wertzuschätzen.

\section{Literatur}

1. Bessin C, Fuchs-Frohnhofen P. Handlungsanregung „Wertschätzendes Führen“. In: Fuchs-Frohnhofen u. a., Herausgeber. PflegeWert - Wertschätzung erkennen, fördern, erleben. Köln: KDA-Verlag; 2012. S. 272-91.

P. Fuchs-Frohnhofen $(\bowtie)$

Bereich Gesundheitswirtschaft und Pflege, Geschäftsführung,

MA\&T Sell \& Partner GmbH, Würselen, Deutschland 